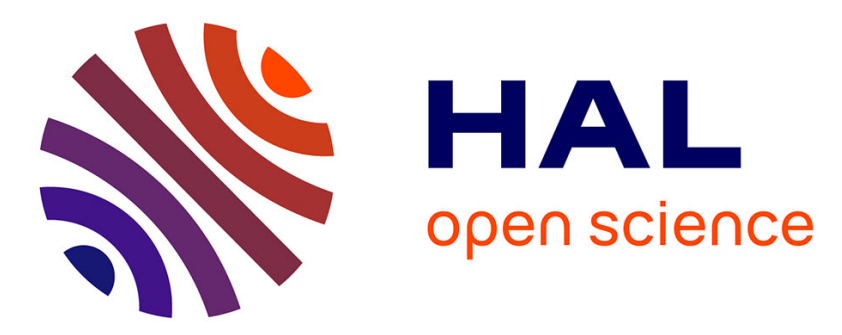

\title{
PIV measurements of an air jet impinging on an open rotor-stator system
}

Thien Nguyen, Julien Pellé, Souad Harmand, Sébastien Poncet

\section{To cite this version:}

Thien Nguyen, Julien Pellé, Souad Harmand, Sébastien Poncet. PIV measurements of an air jet impinging on an open rotor-stator system. Experiments in Fluids, 2012, 53, pp.401-412. 10.1007/s00348012-1298-0 . hal-00822034

\section{HAL Id: hal-00822034 \\ https://hal.science/hal-00822034}

Submitted on 13 May 2013

HAL is a multi-disciplinary open access archive for the deposit and dissemination of scientific research documents, whether they are published or not. The documents may come from teaching and research institutions in France or abroad, or from public or private research centers.
L'archive ouverte pluridisciplinaire HAL, est destinée au dépôt et à la diffusion de documents scientifiques de niveau recherche, publiés ou non, émanant des établissements d'enseignement et de recherche français ou étrangers, des laboratoires publics ou privés. 


\title{
PIV measurements of an air jet impinging on an open rotor-stator system
}

Nguyen T.D. • Pellé J. • Harmand S. • Poncet S.

Received: date / Accepted: date

\begin{abstract}
The current work experimentally investigates the flow characteristics of an air jet impinging on an open rotor-stator system with a low non-dimensional spacing, $G=0.02$, and with a very low aspect ratio, $e / D=0.25$. The rotational Reynolds numbers varied from $0.33 \times 10^{5}$ to $5.32 \times 10^{5}$, while the jet Reynolds numbers ranged from $17.2 \times 10^{3}$ to $43 \times 10^{3}$. Particle image velocimetry (PIV) measurements were taken along the entire disk diameter in three axial planes. From the obtained PIV velocity fields, the flow statistics were computed. A recirculation flow region, which was centered at the impingement point and possessed high turbulence intensities, was observed. Local peaks in root-mean-square fluctuating velocity distributions appeared in the recirculation region and near the periphery, respectively. Proper orthogonal decomposition (POD) analysis was applied to the cases of the jet impinging on the rotor with and without rotation to reveal the coherent structures in the jet region.
\end{abstract}

Keywords open rotor-stator $\cdot$ PIV · impinging jet

\section{Nomenclature}

$\langle$.$\rangle \quad time-averaged operator$

$\nu \quad$ kinematic viscosity of air $\left(\mathrm{m}^{2} / \mathrm{s}\right)$

$\Omega \quad$ rotational speed (rpm)

$\omega \quad$ rotational speed $(\mathrm{rad} / \mathrm{s})$

$C_{w} \quad=Q /\left(\nu R_{e x t}\right)$, mass flow rate coefficient

$G \quad=e / R_{e x t}$, non-dimensional spacing

Nguyen T.D.(凶) · Pellé J. · Harmand S.

Université Lille Nord de France, F-59000 Lille, France

UVHC, TEMPO, F-59313 Valenciennes, France

E-mail: DuyThien.Nguyen@univ-valenciennes.fr

Poncet S.

M2P2, UMR 6181 CNRS-Aix-Marseille Université

13451 Marseille, France 


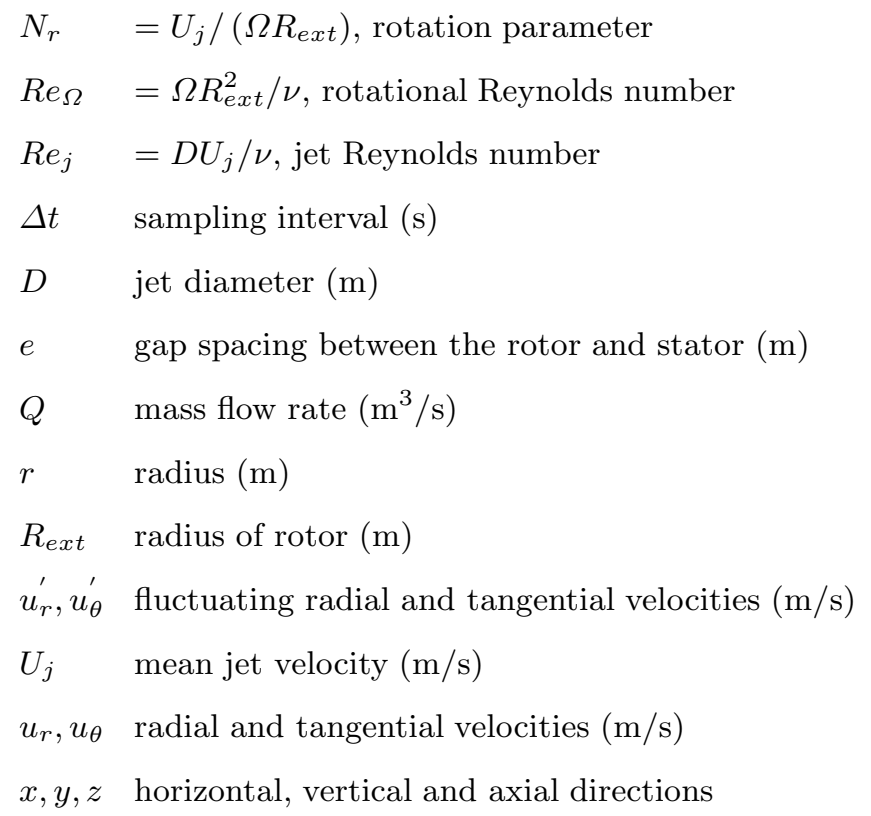

\section{Introduction}

An effective cooling system is nearly always required for any rotating machinery. In this paper, we specially consider the rotating machinery, which consists of rotating disk systems. Such systems are commonly seen in many engineering applications, such as alternators, gas turbine engines, car break disks, computer disk drives, rotational air cleaners, evaporators, etc. The rotating disk systems used in these applications usually are the cases of flows between a rotating disk (rotor) and a stationary disk (stator), closed cavities of gas turbines formed by two disks and cylindrical surfaces, a rotating disk in an infinite resting fluid, co-rotating or counter-rotating parallel disks with and without radial, axial through flows in a gap between them, etc. A specific engineering application of the rotating disk systems considered in this study is the alternator that can be found in modern wind generators of the power production industry. Wind power has recently become as a renewable or "green" source of energy to alter fossil fuels but efforts are required to reduce the costs of wind generators and increase the energy generated per aerogenerator. Attempting to increase the generator efficiency, industrialists have developed the modern wind generators, which normally consist of discoidal rotor-stator systems. These systems do not use gears allowing the generators to operate at low rotational speeds and reducing energy losses. The reference case chosen for this study was a wind generator that has been established in the north of France. This wind generator has a radius of $3 \mathrm{~m}$ and is able to deliver $750 \mathrm{~kW}$ with a maximum rotational speed of $25 \mathrm{rpm}$. With an aim toward more power production, engineers need to solve the problem of ineffective cooling due to high electrical losses dissipated for a relative low rotational speed. An improvement on the cooling of discoidal rotor-stator alternators could be obtained by using air jet impingement.

Air jet impingement is an attractive cooling mechanism that is widely used in rotating disk systems. There have been numerous studies on a jet impinging on a rotating surface. For example, by using a laser Doppler velocimetric method, Brodersen and Metzger (1992) studied the detailed structures of the turbulent velocity fields resulting from the interaction between a jet impinging on a rotating disk. They 
found that the jet penetrated very deeply into the wall boundary layer and the jet expanded against the direction of disk rotation, even at locations close to the disk. Using a hot-wire anemometer to measure mean velocity distributions and Reynolds stresses, Itoh and Okada (1998) investigated the effects of wall rotation for a radial wall jet impinging on a rotating disk. In this work, they studied the flow with a rotational Reynolds number $R_{\Omega}=6 \times 10^{5}$, a non-dimensional gap spacing $G=e / R_{e x t}=0.01$ and an aspect ratio $e / D=1.5$. The authors found that the radial component of turbulence intensity was scarcely affected by the disk rotation, while the integral length scale of the turbulence was significantly reduced by the effects of rotation. Owen and Rogers (1989) experimentally studied the flow and heat transfer for a turbulent flow in a rotor-stator configuration with a central opening and no airflow imposed in the air gap. In their work, the authors showed that boundary layers developed on the rotor and the stator at values of $G$ between 0.01 and 0.06, then explained the distributions of mean Nusselt number on the free disk. Recently, Poncet et al (2005) studied the turbulent flow inside an annular rotor-stator cavity, for which the experimental configuration was $G=0.036$, with and without a superimposed throughflow. Their numerical calculations were validated by two-component laser Doppler anemometer (LDA) measurements. However, those studies were done on a confined rotor-stator configuration with an axial outlet. In order to introduce a new cooling solution for practical applications of rotating disk systems, Pellé and Harmand (2009) experimentally studied the local heat transfer between the rotor surface and the air gap of an open rotor-stator system in which an air jet passed through a hole in the stator and impinged on the rotor. The authors showed the advantage of adding a jet impinging on the center of the rotor, which was in a significant increase in the heat transfer near the jet region. However, the flow characteristic inside such an open rotor-stator configuration is not fully understood due to the lack of available data.

The objective of this paper is to investigate the flow characteristics of an air jet impinging on an open rotor-stator configuration with a low non-dimensional spacing $(G=0.02)$ and a very low aspect ratio $(e / D=0.25)$. Rotational Reynolds numbers varied from $0.33 \times 10^{5}$ to $5.32 \times 10^{5}$, while jet Reynolds numbers ranged from $17.2 \times 10^{3}$ to $43 \times 10^{3}$. In this study, we obtained particle image velocimetry (PIV) measurements at three axial planes for the entire disk diameter. The open rotor-stator system and PIV experimental setup are presented in section 2 In section 3 the obtained PIV results are compared with those obtained by LDA measurements and numerical simulation reported in Poncet et al (2005). In order to reveal the most dominant flow structures, which play an important role in the flow dynamics and heat transfer, proper orthogonal decomposition (POD) is applied to the PIV velocity fields obtained near the jet. In section 4 a brief review of the POD technique and the results from the flow decomposition are presented.

\section{Experimental study}

\subsection{Rotor-stator experimental configuration}

Figure 1 shows the experimental configuration of the jet impinging on the open rotor-stator system. The $x$ and $y$ coordinates respectively represent the horizontal and vertical directions in the PIV measurement plane, which is perpendicular to the jet axis denoted by $z$. The origin of the coordinate system is at the 


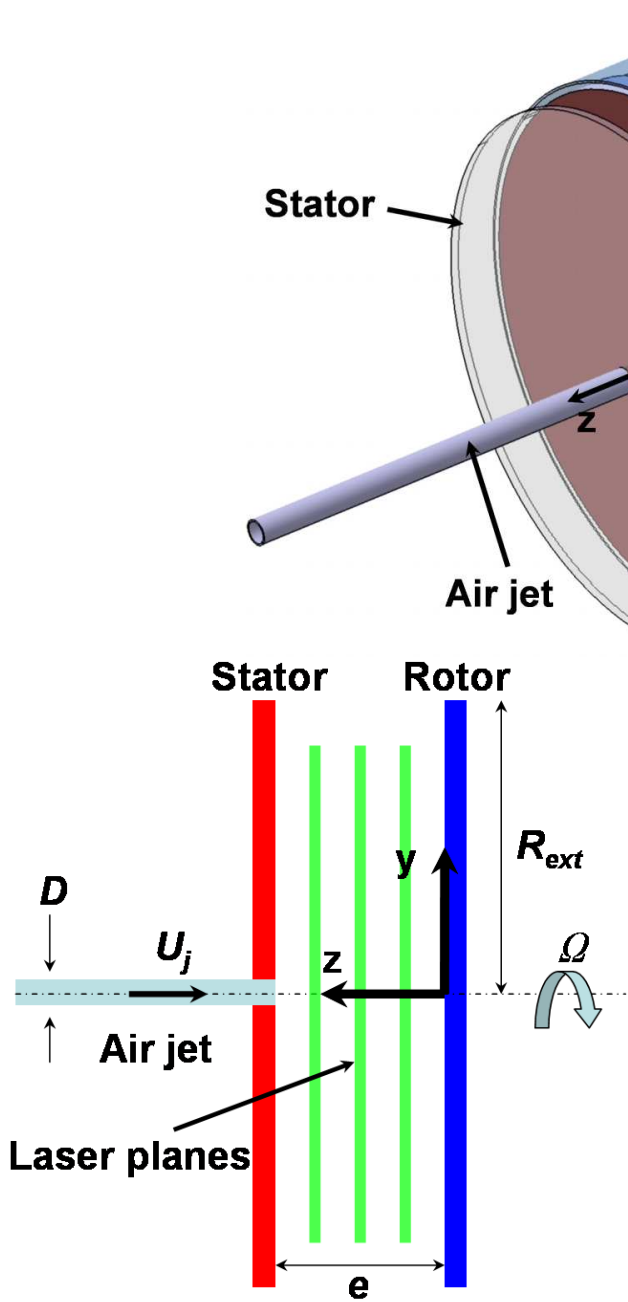

Side view

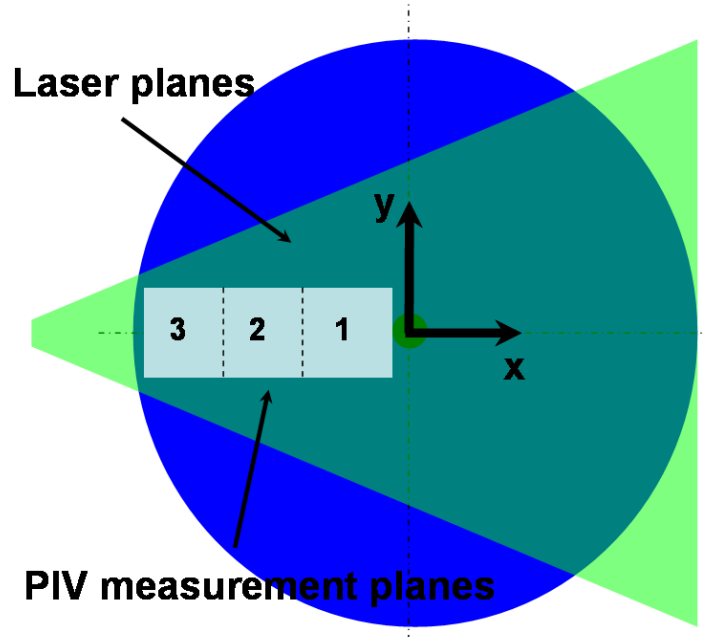

Front view

Fig. 1 Open rotor-stator experimental rig (above) and PIV experimental setup (below).

center of the rotor. We note here three parameters that govern the characteristics of the flow: the nondimensional spacing $G$, the rotational Reynolds number $R e_{\Omega}$ and the jet Reynolds number $R e_{j}$, defined as

$$
\begin{gathered}
G=e / R_{e x t} \\
R e_{\Omega}=\Omega R_{e x t}^{2} / \nu, \\
R e_{j}=D U_{j} / \nu
\end{gathered}
$$

where $e$ is the gap spacing between the rotor and stator, $\nu$ is the kinematic viscosity of air, $R_{\text {ext }}$ is the radius of the rotor $\left(R_{e x t}=310 \mathrm{~mm}\right), D$ is the diameter of the impinging jet $(D=26 \mathrm{~mm})$ and $U_{j}$ is the mean air jet velocity. In the present experiment, the rotating disk (rotor) was connected to a rotating shaft and its rotational speed was controlled by a frequency regulator. Three rotational speeds of $\Omega=50,400$ and 800 rpm, corresponding to the rotational Reynolds numbers of $\operatorname{Re}_{\Omega}=0.33 \times 10^{5}, 2.66 \times 10^{5}$ and $5.32 \times 10^{5}$, respectively, were considered. The stationary disk (stator), which was constructed of transparent plexiglass with a thickness of $10 \mathrm{~mm}$ and a radius of $320 \mathrm{~mm}$, was placed at a distance of $e=6.5 \mathrm{~mm}$ from the rotor, yielding a non-dimensional spacing $G=0.02$. A hole was made at the center of the stator and was connected 
to a centrifugal blower to form the air jet. Air from the blower flowed through the hole in the stator, after which the air flow impinged on the rotating surface of the rotor and interacted with the boundary layer, and then rapidly convected along the radial direction. The mean air jet velocities were $U_{j}=10,15$ and 25 $\mathrm{m} / \mathrm{s}$, yielding jet Reynolds numbers of $R e_{j}=17.2 \times 10^{3}, 25.8 \times 10^{3}$ and $43 \times 10^{3}$, respectively.

\subsection{PIV experimental setup}

The PIV system consisted of a New Wave Nd:YAG laser (TSI Inc.) with an articulated light arm, a digital charge couple device (CCD) camera, a synchroniser and a computer. The Nikkor camera lens had a 105-mm focal length and an f/5.6 aperture. Each laser beam of the double-pulsed laser was capable of $200 \mathrm{~mJ} / \mathrm{pulse}$ at a wavelength of $532 \mathrm{~nm}$. These beams were adjusted by a cylindrical and a spherical lens to form a 1-mm-thick laser sheet. In the rotor-stator experiment, the impinging jet possessed a three-dimensional structure that produced particle displacements normal to the light sheet. These displacements make the out-of-plane lost of pairs significant such that the signal strength of the correlation peak obtained from crosscorrelation calculations strongly diminishes. As a result, the possibility of seeking a valid peak reduces. We have employed two methods suggested by Raffel et al (2007) to overcome this difficulty. These methods were to choose an appropriate thickness of the laser sheet and the time interval between the recordings to accommodate the out-of-plane motion for a given pulse delay. The laser sheet thickness was therefore optimised to be thin enough to generate adequate particle intensity but thick enough to reduce the loss of particle image pairs. An olive oil droplet generator (TSI 9307) generated particles with a mean diameter of $1 \mu \mathrm{m}$ to seed the inlet of the centrifugal blower. The TSI PowerView Plus 4MP camera with a resolution of $2048 \times 2048$ pixels and a pixel size of $7.4 \times 7.4 \mu \mathrm{m}^{2}$ captured PIV images and directly sent them to the computer. A LaserPulse (TSI Model 610035) synchroniser controlled the synchronisation between the lasers and camera. Depending on the jet velocity and the rotational speed, the time interval between the first and the second exposures varied from 15 to $150 \mu \mathrm{s}$, yielding maximum particle displacements of 6 pixels.

In this flow configuration, we performed PIV measurements along the axial direction in three planes defined by $z_{1} / e=0.23$ (near the rotor), $z_{2} / e=0.53$ (the middle plane) and $z_{3} / e=0.84$ (near the stator). At each axial plane, PIV images were separately captured at three different regions with similar flow conditions to cover the rotor radius. Specifically, the PIV measurements of regions 1, 2 and 3 covered the flow regions in the $x-y$ plane with $x /\left(-R_{e x t}\right)$ ranging from 0.04 to $0.37,0.38$ to 0.71 , and 0.72 to 1 , respectively. For the specific values of $R e_{\Omega}$ and $R e_{j}$, a sequence of 500 image pairs was recorded during each run with a sampling rate of $1 \mathrm{~Hz}$. We denote by $\Delta t$ the corresponding sampling interval of $1 \mathrm{~s}$. For the region near the jet, nondimensionalised by the mean jet velocity $U_{j}$ and by the jet diameter $D$, this interval becomes $\Delta t \times U_{j} / D$, with values ranging from 380 to 960 . For the outer region, the sampling interval can be nondimensionalised by the rotational speed $\Omega$ and the jet diameter $D$, which is the largest-scale structure of the flow, or the rotor radius $R_{\text {ext }}$. The former interval is $\Delta t \times \frac{\pi \Omega R_{e x t}}{30 D}$, ranging from 62 to 998 , and

the latter interval is $\Delta t \times \frac{\pi \Omega R_{e x t}}{30 R_{e x t}}$, ranging from 5.2 to 83 . In all cases, the nondimensionalised sampling interval shows that the particles travel a very long distance compared to the largest-scale structure of the 
flow or the rotor radius. Therefore, the velocity fields obtained from the PIV image pairs are statistically independent.

Image acquisition and image processing were performed with TSI Insight TM 3G software (version 9.1). The seeding density was adjusted to yield an average of 12 particle images in an interrogation window of $32 \times 32$ pixels. We analysed the digital PIV images by using a recursive Nyquist rectangular grid algorithm with two iterations. This algorithm started with an interrogation window of $64 \times 64$ pixels and ended with an interrogation window of $32 \times 32$ pixels. Both iterations had a $50 \%$ window overlap, yielding the final number of $110 \times 110$ vectors. The first iteration initially computed the particle displacements, which were then used to shift the interrogation window in the second iteration. In both of the iterations, vectors were computed from the correlation map with a Gaussian peak fit (Raffel et al, 2007) for sub-pixel accuracy, and were validated by the signal-to-noise ratio of 1.5. The final step was to apply a median filter (Westerweel, 1994) and a standard deviation filter to remove spurious vectors, and then fill in the blanks by interpolation.

For all the tests, the percentage of bad vectors, which was calculated as the average over the number of PIV velocity fields, was about $2 \%$. The uncertainties in the PIV velocity measurements were estimated to be less than $9 \%$ of the mean jet velocity in region 1 and typically less than $4 \%$ and $6 \%$ in regions 2 and 3, respectively. The corresponding overall standard deviations of velocity in region 1,2 and 3 were $6 \%, 2 \%$ and $4 \%$, respectively, of the mean jet velocity. The uncertainty calculation was based on the error analysis developed by Coleman and Steele (1995); this error analysis was previously applied to PIV velocity measurements by Gui et al (2001) and by Murray and Ukeiley (2007).

\section{Results from PIV measurements}

3.1 Comparisons with LDA measurement and RSM simulation

In this section, we first compare the PIV results with those from the two-component laser Doppler anemometer (LDA) measurements and numerical simulation by Poncet et al (2005). This simulation was based on one-point statistical modelling using a low-Reynolds-number, second-order, full stress transport closure, or Reynolds Stress Model (RSM). Note that these experimental and numerical configurations are the most currently available and compatible with our test configuration. The LDA measurements and RSM simulation were done with a confined rotor-stator configuration and an axial outlet. In both the experiment and simulation, a central hub was connected to the rotor and a fixed shroud enclosed the space between rotor and stator. The parameters of the PIV measurements, LDA experiments and RSM simulation are listed in table[1 The mass flow rate coefficient is defined by $C_{w}=Q /\left(\nu R_{e x t}\right)$. In our experiment, this coefficient was $C_{w}=-2830$, corresponding to the maximum jet velocity $U_{j}=25 \mathrm{~m} / \mathrm{s}$. The rotation parameter, defined by $N_{r}=U_{j} /\left(\Omega R_{e x t}\right)$, and the ratio of $\Omega D / U_{j}$ are almost equal for the PIV and LDA experiments and RSM simulation.

The radial $u_{r}$ and tangential $u_{\theta}$ velocity components measured at three radial locations: $r / R_{\text {ext }}=$ $0.44,0.56$, and 0.8 , were considered in the comparisons among the PIV and LDA measurements and the RSM simulation. In the PIV results, statistics of $u_{r}$ and $u_{\theta}$ were computed and spatially averaged along 

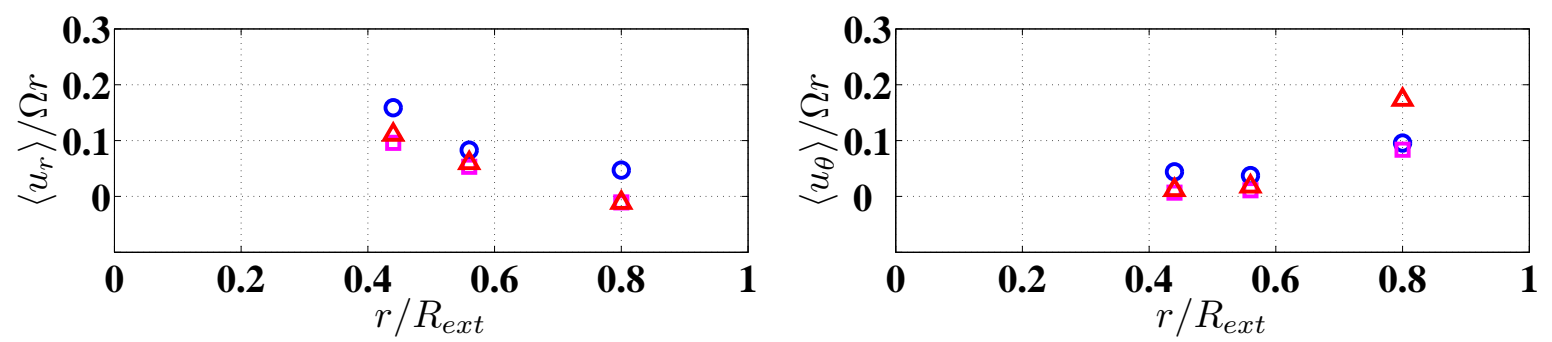

(a) Near the stator
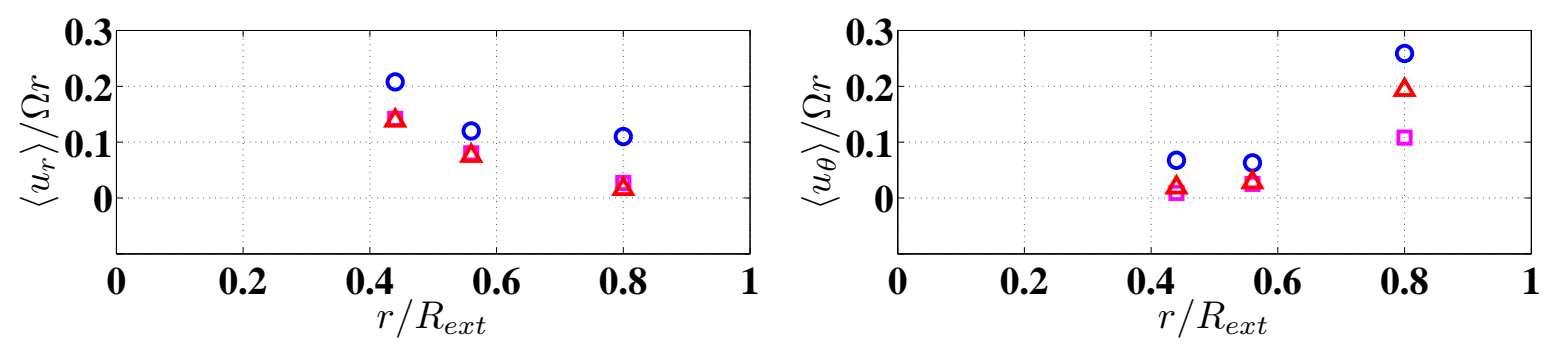

(b) Middle plane
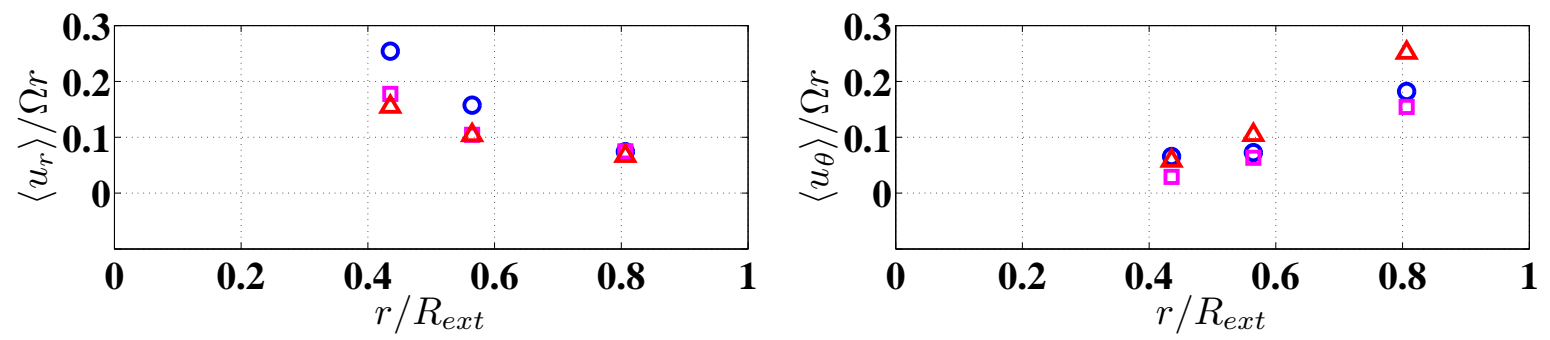

(c) Near the rotor

Fig. 2 Comparison of the mean velocity obtained by PIV ( $\bigcirc)$, LDA $(\square)$ and RSM $(\triangle)$ at different axial planes, (a) near the stator, (b) middle plane, (c) near the rotor.

these three radii. Figure 2 shows the comparisons of the mean radial $\left\langle u_{r}\right\rangle$ and tangential $\left\langle u_{\theta}\right\rangle$ velocities obtained from the three axial planes: near the stator, the middle plane and near the rotor. Figure 3 shows the turbulence intensities $\sqrt{\left\langle u_{r}^{\prime 2}\right\rangle}, \sqrt{\left\langle u_{\theta}^{\prime 2}\right\rangle}$ and $\left\langle u_{r}^{\prime} u_{\theta}^{\prime}\right\rangle$ of the PIV and LDA measurements and the RSM simulation. These values were nondimensionalised by the local rotational speed, $\Omega r$, at the same radial points. Relative differences between the statistics, i.e. mean velocity and Reynolds stress components, of PIV and LDA measurements, and those of PIV measurement and RSM simulation were calculated as

$$
\delta=\frac{1}{3} \sum_{z_{i}=1}^{3}\left|\frac{S_{z_{i}, P I V}-S_{z_{i}, L D A / R S M}}{S_{z_{i}, P I V}}\right|,
$$

where $S_{z i, P I V}$ is the statistics of PIV measurement, $S_{z i, L D A / R S M}$ is the statistics of LDA measurement or RSM simulation, respectively, and $z_{i}$ stands for the measurement planes in the axial direction. Figure 4 shows the calculated relative differences from the statistics of the PIV and LDA measurements and RSM

Table 1 Parameters of PIV, LDA experiments and RSM simulation.

\begin{tabular}{|c|c|c|c|c|c|}
\hline & $R e_{\Omega}$ & $C_{w}$ & $N_{r}$ & $G$ & Configuration \\
\hline PIV & $5.3 \times 10^{5}$ & -2830 & 0.0053 & 0.02 & Open rotor-stator \\
\hline LDA (Poncet et al, 2005) & $10^{6}$ & -5929 & 0.0059 & 0.036 & Shrouded rotor-stator \\
\hline RSM (Poncet et al, 2005) & $10^{6}$ & -5929 & 0.0059 & 0.036 & Shrouded rotor-stator \\
\hline
\end{tabular}



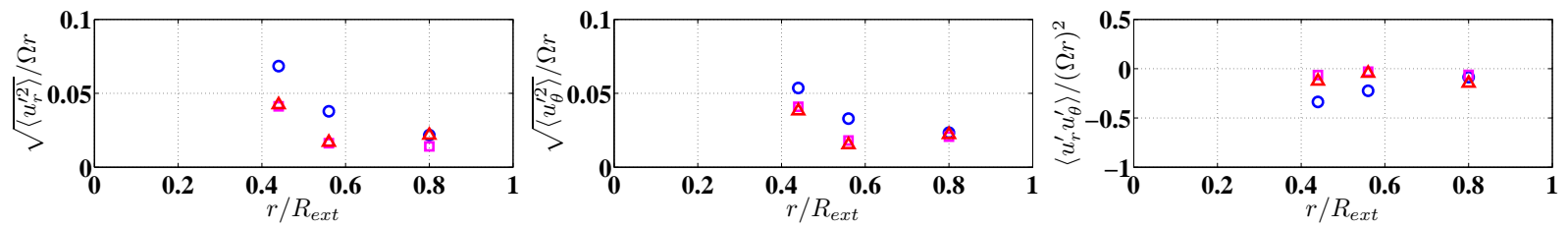

(a) Near the stator
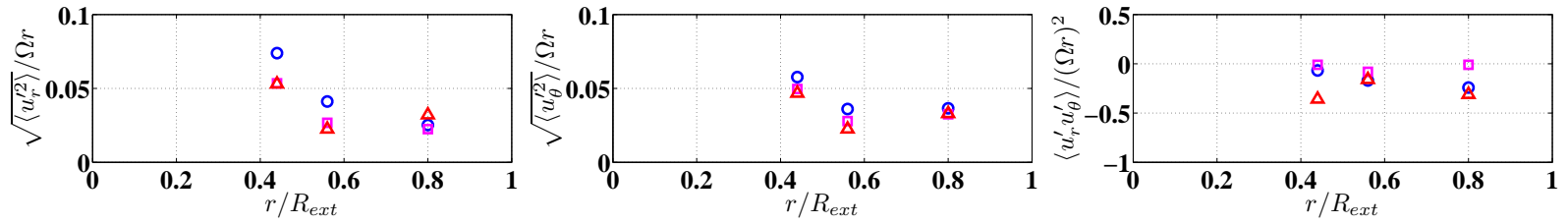

(b) Middle plane
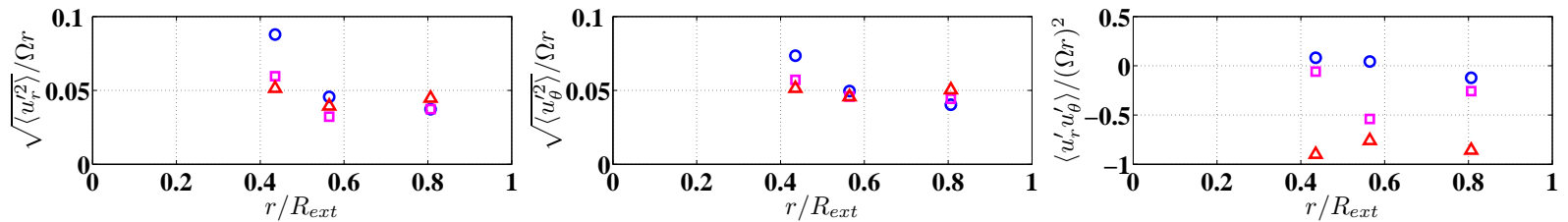

(c) Near the rotor

Fig. 3 Comparison of the turbulence intensities obtained by PIV (O ), LDA ( $\square)$ and RSM $(\triangle)$ at different axial planes, (a) near the stator, (b) middle plane, (c) near the rotor

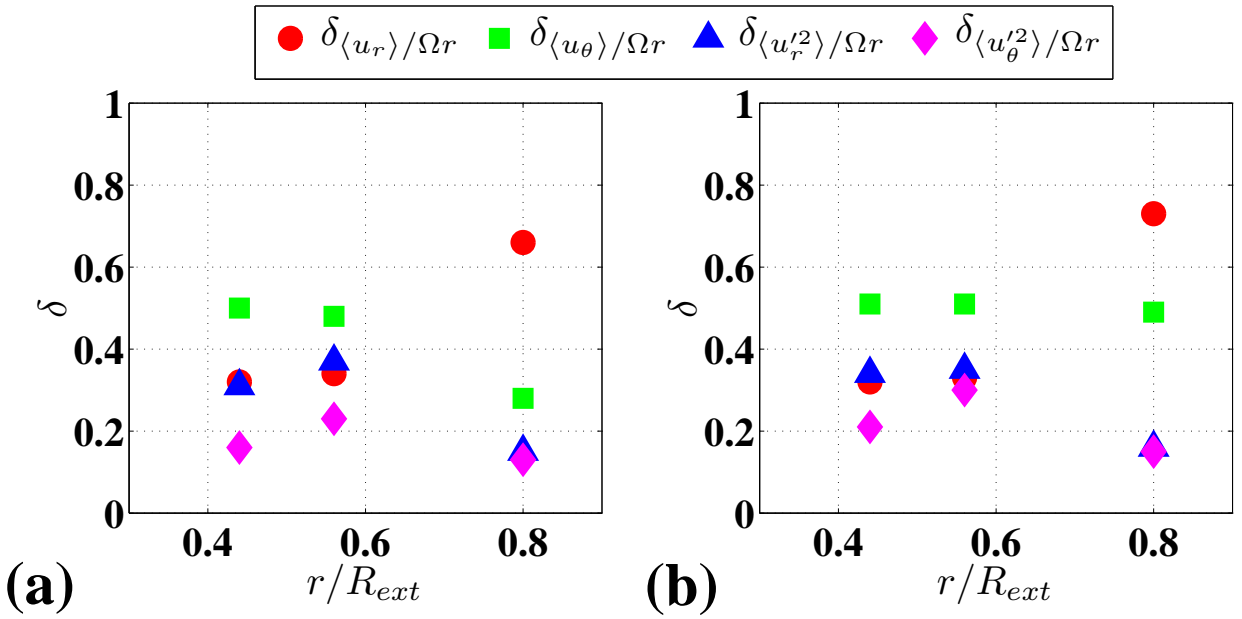

Fig. 4 Relative differences between the statistics of (a) PIV and LDA, (b) PIV and RSM.

simulation. Relative differences corresponding to the $\left\langle u_{r}^{\prime} u_{\theta}^{\prime}\right\rangle$ component are not shown due to the major discrepancies among the PIV, LDA and RSM results obtained near the rotor. In the mid-radius region, the flow is centrifugal yielding $\left\langle u_{r}\right\rangle / \Omega r>0$ for all the axial planes and $\left\langle u_{\theta}\right\rangle / \Omega r \simeq 0$ near the stator. At the radial location $r / R=0.44$, a reason for the relative differences of $\left\langle u_{\theta}\right\rangle$ could be the averaged-volume property of the PIV technique, which is characterised by the laser thickness and the interrogation window size $\left(1 \times 2 \times 2 \mathrm{~mm}^{3}\right)$. This volume is not small enough compared to the boundary layer thickness and the small gap between the rotor and the stator. In the outer region $r / R>0.7$, Poncet et al (2005) showed two boundary layers separated by a rotating core. The flow was centripetal near the stator, yielding $\left\langle u_{r}\right\rangle / \Omega r<0$, and was centrifugal near the rotor, yielding $\left\langle u_{r}\right\rangle / \Omega r>0$. Such flow structure, however, was not observed in our PIV experiments without the shroud for $R e_{\Omega}=5.3 \times 10^{5}$ and $R e_{j}=43 \times 10^{3}$. The fixed shroud then 
probably yielded a significant difference for the $\left\langle u_{r}\right\rangle / \Omega r$ at the radial location $r / R=0.8$. It is seen that the shroud yielded a weak difference to the $\left\langle u_{r}^{\prime 2}\right\rangle$ and $\left\langle u_{\theta}^{\prime 2}\right\rangle$ components at the outer radius, while the relative differences of these components at the inner radius are greater.

\subsection{Mean flow and turbulence statistics}

Figure 5 shows the mean PIV velocity fields obtained at the middle plane with $R e_{j}=17.2 \times 10^{3}$ and $R e_{\Omega}=0,0.33 \times 10^{5}, 2.66 \times 10^{5}$ and $5.32 \times 10^{5}$. The velocity vectors are nondimensionalised by the mean jet velocity $U_{j}$, which is also overlaid on the right hand side of these figures.

Flow visualisation studies by Baydar and Ozmen (2006) demonstrated that for a jet impinging on a plate with an aspect ratio $e / D=1$ and a jet Reynolds number $R e_{j}=4.8 \times 10^{3}$, a strong recirculation zone occurred in the confined region around the jet axis. Such a recirculation zone did not appear in the unconfined jet in this experiment. Although in the current PIV experiments, measurements were performed in planes normal to the axial direction, the mean velocity vectors in figure 5 ( $a$ ) show that no recirculation formed in the region between the rotor and stator (note the very low aspect ratio - $e / D=0.25$ - compared to $e / D=1$ in Baydar and Ozmen (2006)). This figure also shows that the impinging jet flow without rotation was outward across the entire radius. In contrast, figures $\mathbf{5}(b, c, d)$ illustrate that recirculation zones occurred and was centered on the jet axis. The effect of the rotation is clearly observed near the periphery when the rotational speed increases. The flow is radial over nearly the whole rotor radius for $\operatorname{Re}_{\Omega}=0.33 \times 10^{5}$ but becomes tangential towards the periphery, starting from $r / R_{e x t}=0.6$ for $R_{\Omega}=2.66 \times 10^{5}$ and from $r / R_{\text {ext }}=0.4$ for $\operatorname{Re}_{\Omega}=5.32 \times 10^{5}$. Because the thickness of the boundary layers that develop on the rotor and stator increases in the radial direction, the flow is gradually dominated by the rotation at the larger radii. In our experiments, the mean velocity fields do not show an inflow zone near the outer radius of the stator as found in Boutarfa and Harmand (2005). It should be noted that the ratio between the radius of the central opening in the stator and the rotor radius is 0.89 in Boutarfa and Harmand (2005), while it is much smaller (0.08) in our experiment.

Considering the effects of the stator, Owen and Rogers (1989) related the rotational Reynolds number $R e_{\Omega}$ and a limiting value of the non-dimensional spacing $G$ as

$$
G_{l i m}=1.05 R e_{\Omega}^{-0.2}
$$

below which the stator yields effects to the flow structure and the heat transfer. In our study, the rotational Reynolds numbers $R_{\Omega}$ varied from $0.33 \times 10^{5}$ to $5.32 \times 10^{5}$ yielding $G_{l i m}=0.07$ and 0.13 . The nondimensional gap spacing $G=0.02$ indicates that the presence of the stator is expected to have a strong influence on the flow structures. Figure 6 shows the differences among the nondimensionalised radial velocity $\left\langle u_{r}\right\rangle / U_{j}$ and root-mean-square (r.m.s) fluctuating velocity $\sqrt{\left\langle u_{r}^{\prime 2}\right\rangle} / U_{j}$ obtained from the current PIV results with $R e_{j}=25.8 \times 10^{3}$, and those obtained from the LDA measurements of Minagawa and Obi (2004) and the Elliptic-Blending Reynolds Stress Model (EB-RSM) of Manceau et al (2009) with $R e_{j}=14.5 \times 10^{3}$. The $\mathrm{PIV}$ and LDA experiments have the same rotation rate $\omega D / U_{j}=0.15$ while the rotation rate of EB-RSM simulation is slightly smaller, $\omega D / U_{j}=0.12$. It is necessary to note that the LDA and EB-RSM results were 

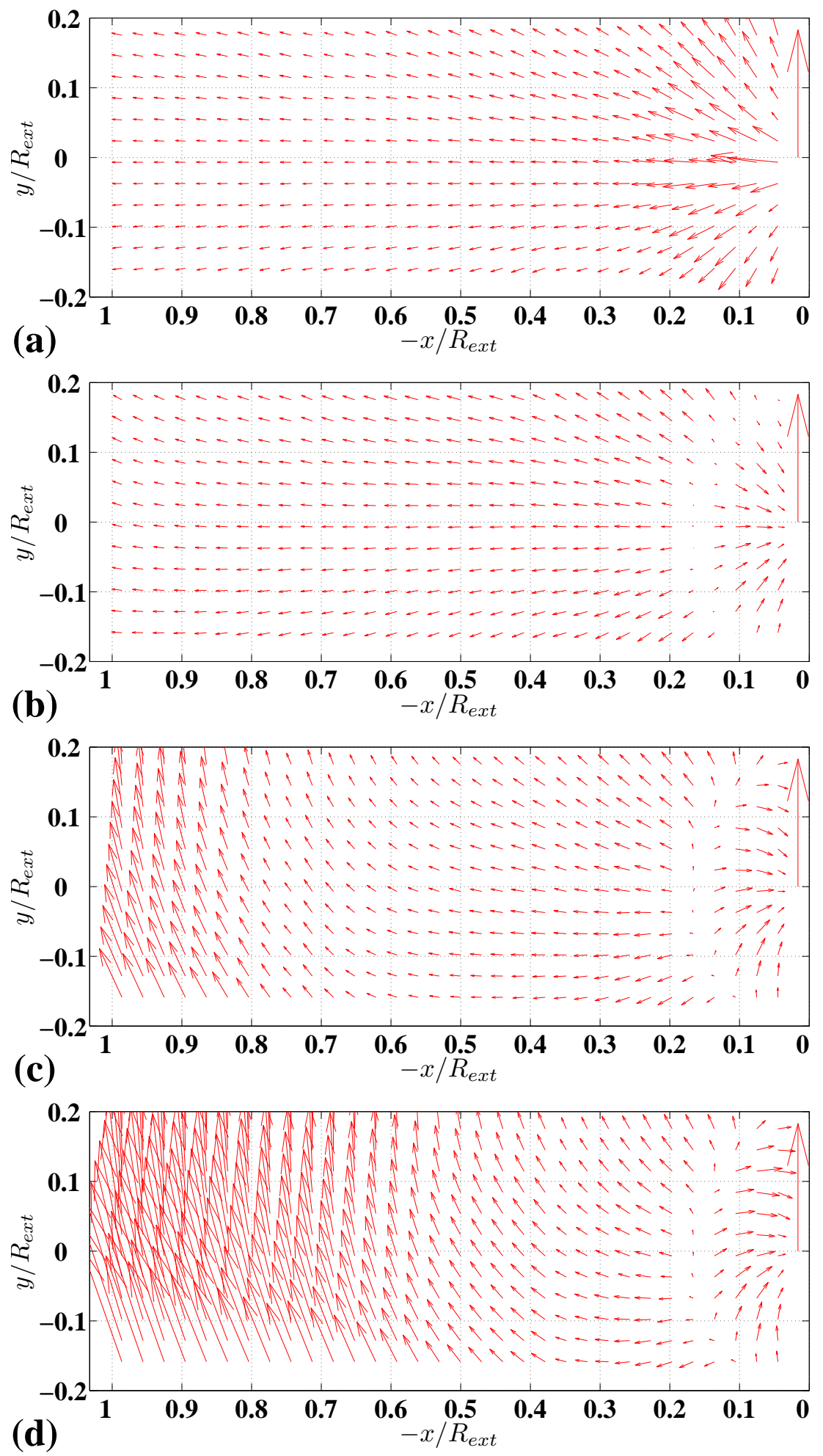

Fig. 5 Nondimensionalised mean PIV velocity fields of the jet impinging on the open rotor-stator, $R e_{j}=17.2 \times 10^{3},(a)$ $R e_{\Omega}=0$, (b) $R e_{\Omega}=0.33 \times 10^{5}$, (c) $R e_{\Omega}=2.66 \times 10^{5}$, (d) $R e_{\Omega}=5.32 \times 10^{5}$. Velocity vectors are de-sampled for better visibility.

obtained for the case of impinging jet without a stator. The presence of the stator has a strong influence on the flow structures as shown in figure 6(a) by the negative values of $\left\langle u_{r}\right\rangle / U_{j}$, which reveal a recirculation 

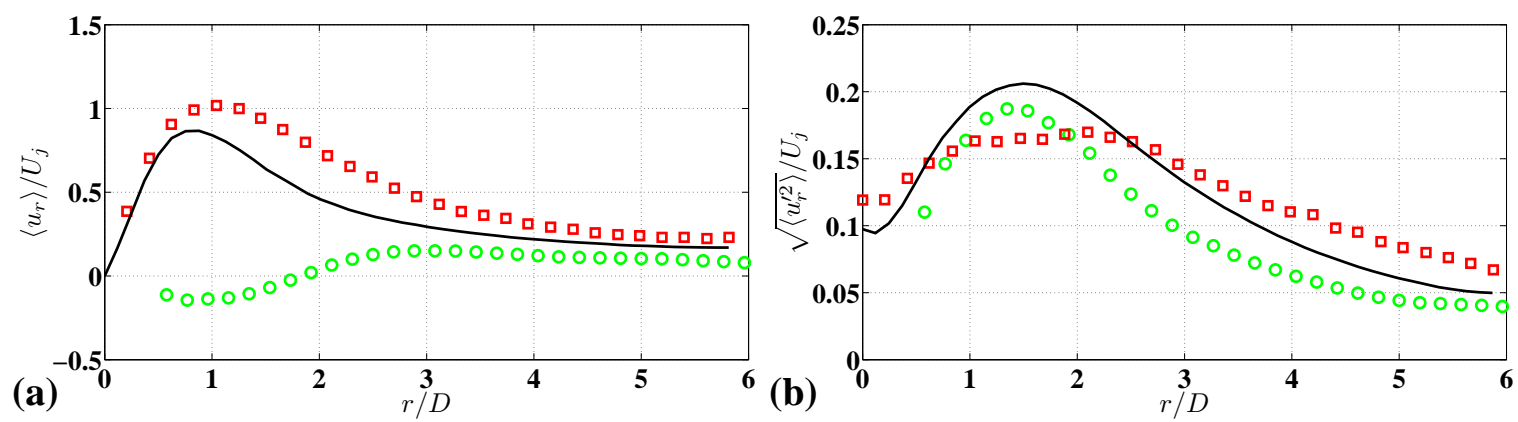

Fig. 6 Comparisons of (a) the mean radial velocity $\left\langle u_{r}\right\rangle$, and $(b)$ r.m.s fluctuating radial velocity $\sqrt{\left\langle u_{r}^{\prime 2}\right\rangle}$ obtained by PIV (O ), LDA (口, Minagawa and Obi (2004)) and EB-RSM (—, Manceau et al (2009)). The LDA and EB-RSM results were obtained for the case of impinging jet without a stator.
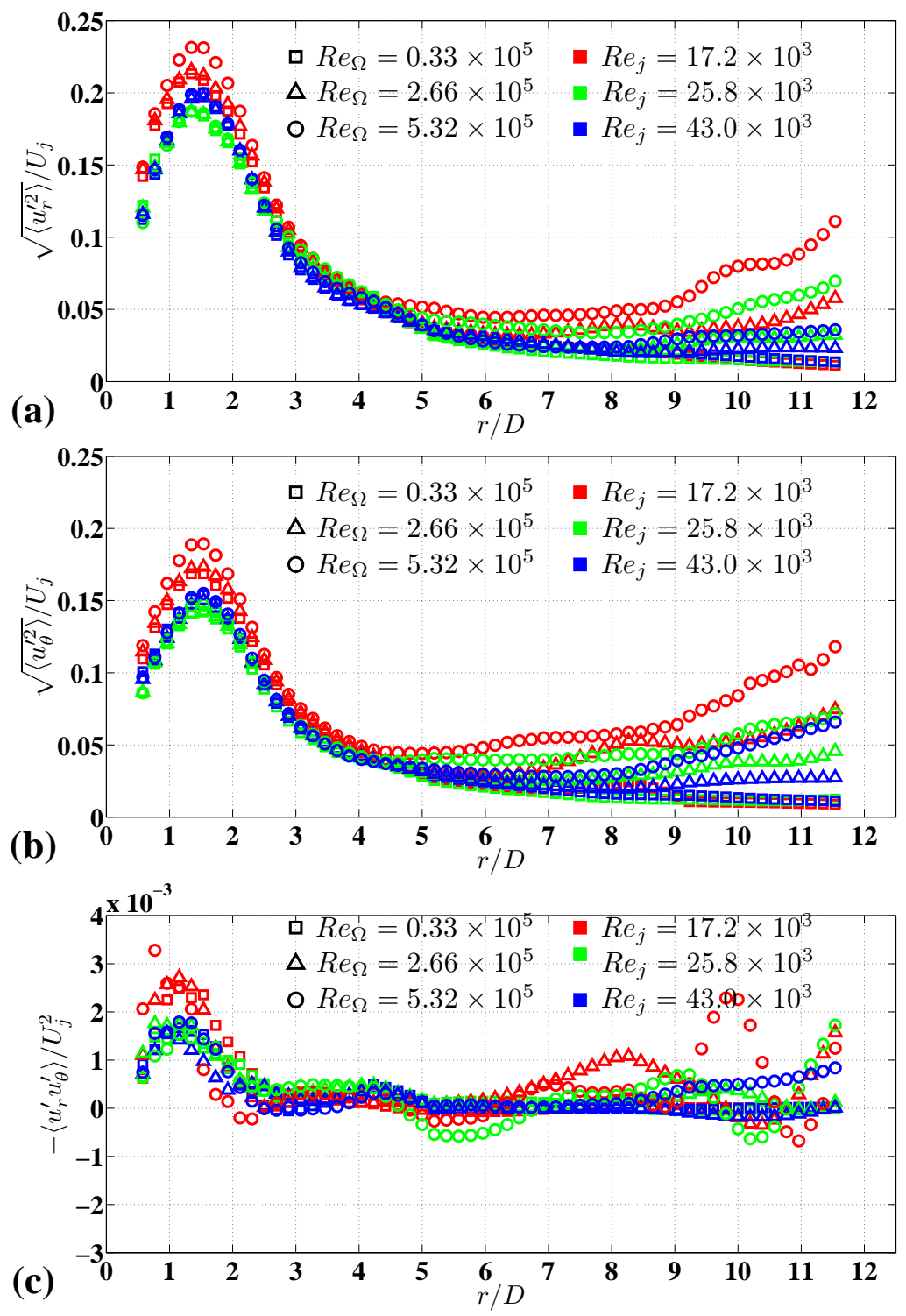

Fig. 7 Nondimensionalised r.m.s fluctuating velocities obtained by PIV measurements near the rotor with various rotational speeds and jet velocities, (a) $\sqrt{\left\langle u_{r}^{\prime 2}\right\rangle} / U_{j}$, (b) $\sqrt{\left\langle u_{\theta}^{\prime 2}\right\rangle} / U_{j}$, (c) $\left\langle-u_{r}^{\prime} u_{\theta}^{\prime}\right\rangle / U_{j}^{2}$.

region near the impingement. Such flow structure, which is not visible in the LDA and EB-RSM results, also causes the differences in radial distributions of $\sqrt{\left\langle u_{r}^{\prime 2}\right\rangle} / U_{j}$ as illustrated in figure $6(b)$. 

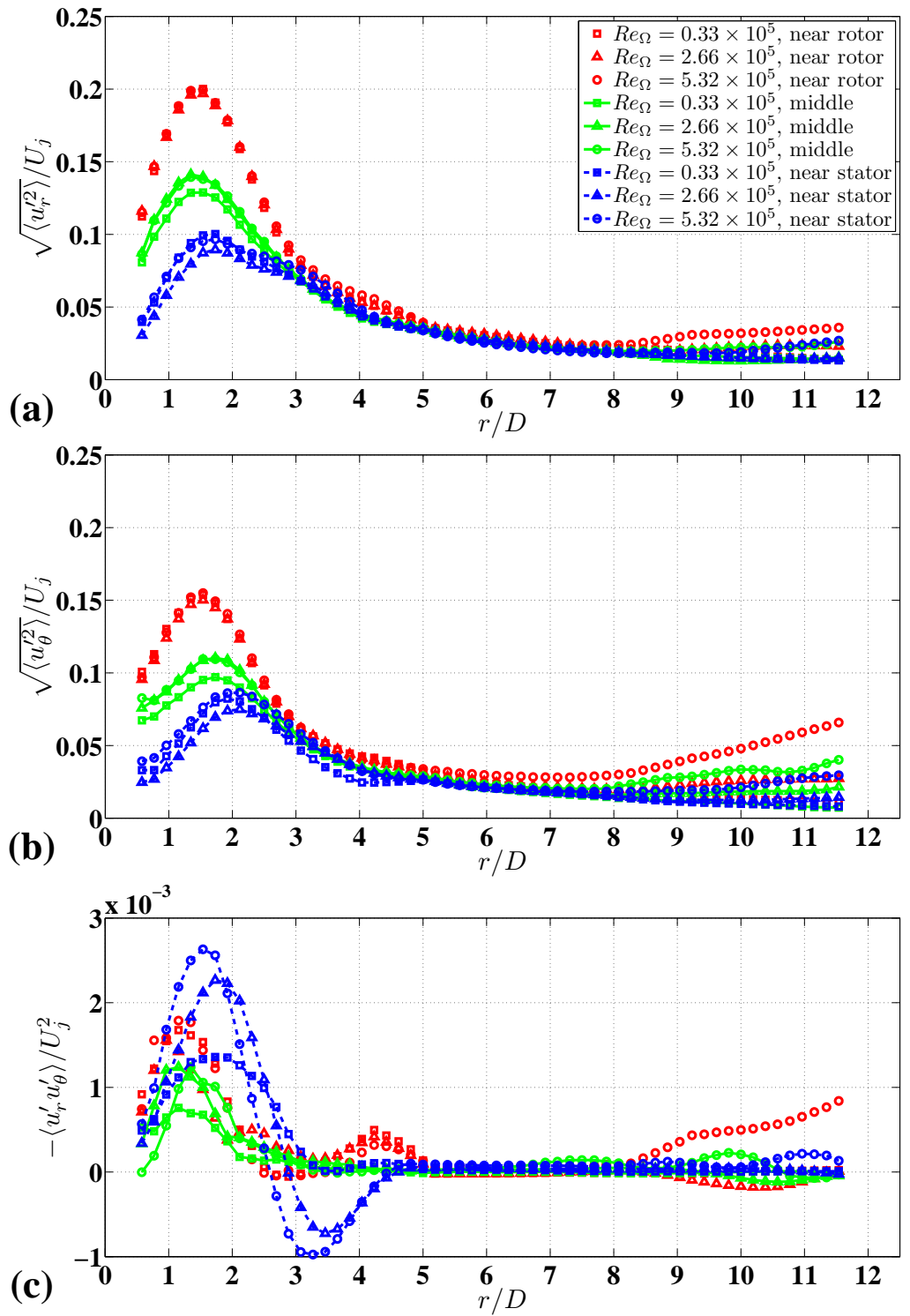

Fig. 8 Nondimensionalised r.m.s fluctuating velocities obtained by PIV measurements at $R e_{j}=43 \times 10^{3}$ with various rotational speeds and different axial planes, (a) $\sqrt{\left\langle u_{r}^{\prime 2}\right\rangle} / U_{j}$, (b) $\sqrt{\left\langle u_{\theta}^{\prime 2}\right\rangle} / U_{j},(c)\left\langle-u_{r}^{\prime} u_{\theta}^{\prime}\right\rangle / U_{j}^{2}$.

Figure 7illustrates the differences among the nondimensionalised r.m.s fluctuating velocities $\sqrt{\left\langle u_{r}^{\prime 2}\right\rangle} / U_{j}$, $\sqrt{\left\langle u_{\theta}^{\prime 2}\right\rangle} / U_{j}$ and the shear component of Reynolds stress $\left\langle-u_{r}^{\prime} u_{\theta}^{\prime}\right\rangle / U_{j}^{2}$ near the rotor, with various values of $R e_{\Omega}$ and $R e_{j}$. Note that the $u_{r}$ and $u_{\theta}$ velocity components were azimuthally averaged along various radial paths in the axial plane $z_{3} / e=0.23$. The maximum angle between two radial paths was about $9^{0}$. The presence of the impinging plane causes a radial flow deflection and accelerates the flow in the radial direction immediately after the stagnation point. A radial wall jet begins to form and creates a wall boundary region downstream from the impingement. The accelerating boundary layer is thinning due to mass conservation. The flow accelerates in the radial direction up to the locations of the peaks of r.m.s fluctuating velocity, and the development of the wall-jet thickness causes the fluid to decelerate. The local peaks of r.m.s fluctuating velocities $\sqrt{\left\langle u_{r}^{\prime 2}\right\rangle} / U_{j}$ and $\sqrt{\left\langle u_{\theta}^{\prime 2}\right\rangle} / U_{i}$ occur at $r / D$ values from 1.5 to 1.6 , owing to the transition to turbulent flow in the wall jet region. Manceau et al (2009) used a non-dimensional parameter $r_{0} \omega / U_{j}$, which indicated the relative magnitude of the centrifugal acceleration, to study the effect of rotation. These authors found 
that the influence of rotation was negligible near the impingement region but significant in the wall jet region after $r / D>2.5$. In our work, the influence of rotation can be identified from figure 7 as the deviation of r.m.s fluctuating velocities. Such effect appears in a region away from the impingement, $r / D \geq 4$, and becomes more obvious at a higher rotational speed. Lytle and Webb (1994) showed that for aspect ratios $e / D<1$, the peak in the velocity fluctuations parallel to the impingement surface corresponded to the peak in the mean heat transfer distribution. Figure 7 also shows that the areas of high r.m.s fluctuating velocity were located in the recirculation region and the peaks in the recirculation region were not strongly affected by an increase of the rotational speed. This observation agrees with the results reported by Itoh and Okada (1998). In contrast, an increase of the jet velocity produced an increase in the background r.m.s fluctuating velocity.

Figure 8 compares the nondimensionalised r.m.s fluctuating velocities $\sqrt{\left\langle u_{r}^{\prime 2}\right\rangle} / U_{j}, \sqrt{\left\langle u_{\theta}^{\prime 2}\right\rangle} / U_{j}$ and the shear component of Reynolds stress $\left\langle-u_{r}^{\prime} u_{\theta}^{\prime}\right\rangle / U_{j}^{2}$ at the middle plane, near the rotor and near the stator. The jet Reynolds number was $R e_{j}=43 \times 10^{3}$ and the rotational Reynolds numbers $R e_{\Omega}$ was varied as indicated. It is seen that after the stagnation point, the r.m.s. fluctuations of the velocity components parallel to the impingement surface rapidly increase and yield the maximum peaks in the wall jet region. The lowest peaks of r.m.s fluctuating velocity occur at the plane near the stator, while the peaks at the middle plane are about $40 \%$ greater. The highest peaks of r.m.s fluctuating velocity correspond to the plane near the rotor, owing to the transition from a laminar to a turbulent boundary layer and the growth of turbulence production caused by the near-wall shear. Another feature is that the positions of the r.m.s fluctuating velocity peaks at the middle plane do not coincide with those obtained at the planes near the rotor and the stator. This is clearly observed in the distributions of $\sqrt{\left\langle u_{\theta}^{\prime 2}\right\rangle} / U_{j}$, c.f. figure 8(b), which show a translation of the peak location from $r / D=1.5$ for the plane near the rotor to $r / D=1.75$ for the middle plane, and $r / D=2.0$ for the plane near the stator. When investigating the flow structures of the confined impinging jet with $e / D=4$, Fitzgerald and Garimella (1998) also found that the turbulence levels near the impinging plane were higher than those near the confinement plane, which corresponded to the rotor and stator planes, respectively, in our experiment. Moreover, these authors discussed that approaching the stator, the turbulence peaks moved radially outward because the turbulence diffused through the boundary layer. Such phenomenon were also observed in Baydar and Ozmen (2006) experiments with a confined impinging air jet of $e / D=1$. Their results showed a radial translation of $\Delta r / D=0.2$ of the locations of the turbulence intensity peaks at different measurement planes. In our experiments, the translation of the peak location is attributed to the turbulence diffusion and a high shear region due to the presence of the rotor that primarily affects the corresponding component $\sqrt{\left\langle u_{\theta}^{\prime 2}\right\rangle} / U_{j}$.

\section{Flow analysis by proper orthogonal decomposition}

\subsection{Proper orthogonal decomposition}

In this section, proper orthogonal decomposition (POD) is applied to the PIV velocity snapshots to reveal the most dominant flow structures, which play an important role in the flow dynamics and heat transfer. 
These velocity fields were obtained near the jet region and corresponded to the cases of $\operatorname{Re}_{j}=25.8 \times 10^{3}$, $R e_{\Omega}=2.66 \times 10^{5}$ and $R e_{\Omega}=0$. Complete descriptions of the direct and snapshot POD techniques are given by Berkooz et al (1993), Holmes et al (1996) and Sirovich and Kirby (1987). In this paper, we apply the snapshot POD to a set of instantaneous flow fields collected from PIV experimental velocity fields. A brief review of the snapshot POD is described here. A correlation matrix is calculated as

$$
C_{i j}=\frac{1}{N} \int \boldsymbol{u}\left(\boldsymbol{x}, t_{i}\right) \cdot \boldsymbol{u}\left(\boldsymbol{x}, t_{j}\right) d \boldsymbol{x}
$$

where $N$ is the number of PIV velocity snapshots. In order to compute the POD basis functions $\boldsymbol{\psi}(\boldsymbol{x})$ and temporal coefficients $\zeta_{k}(t)$, let us first define $\alpha_{k i}$ as

$$
\alpha_{k i}=\frac{v_{i}^{k}}{\sqrt{N \sum_{m=1}^{N} \sum_{r=1}^{N} v_{m}^{k} v_{r}^{k} C_{m r}}}
$$

where $v_{i}^{k}$ is the $i$ th element of the eigenvector $\boldsymbol{v}^{k}$ corresponding to the eigenvalue $\lambda_{k}$ of the correlation matrix $\boldsymbol{C}$. The POD basis functions are then computed as

$$
\boldsymbol{\psi}_{k}(\boldsymbol{x})=\sum_{i=1}^{N} \alpha_{k i} \boldsymbol{u}\left(\boldsymbol{x}, t_{i}\right)
$$

and the temporal coefficients as

$$
\zeta_{k}(t)=\int \boldsymbol{u}(\boldsymbol{x}, t) \cdot \boldsymbol{\psi}_{k}(\boldsymbol{x}) d \boldsymbol{x}=N \sum_{i=1}^{N} \alpha_{k i} C_{i t} .
$$

In our POD calculation, the eigenvalue $\lambda$ associated with each POD mode is proportional to the kinetic energy contained in that mode. The decomposition yields statistically dominant flow structures in the few lowest-order POD modes; these modes capture most of the flow's kinetic energy and are typically associated with large-scale structures. Besides, the POD basis functions computed from these velocity snapshots yield an optimal representation of the flow field in the sense that, for any given number of basis functions, the Hilbert norm of the truncation error between the original velocity data and the projection of the original velocity data onto these basis functions is minimised. The present PIV setup allows us to capture several hundred instantaneous velocity fields. A set of 500 PIV measurements suffices for a POD analysis to reveal the statistically dominant structures of the flow.

Figure 9 shows the energy spectra and cumulative energies of the velocity decomposition. The energy fraction of the first POD mode, which is approximately equal to the average flow, is found to be $54 \%$ of the total energy of the flow for the case $R e_{\Omega}=2.66 \times 10^{5}$. For the case $R e_{\Omega}=0$, the first POD mode captures almost the same total energy of the flow, $53 \%$, while the kinetic energy levels contained in the other loworder modes (modes $2,3,4$, etc) are less than those in the low-order modes for the case $\operatorname{Re}_{\Omega}=2.66 \times 10^{5}$. The kinetic energy levels of the low-order POD modes are less than $1 \%$, resulting in a slow convergence of the cumulative energy; i.e. 90 modes capture about $80 \%$ total kinetic energy of the flow. This indicates that the jet region is highly turbulent and the flow energy is distributed over many small-scale structures.

Figure 10 shows the in-plane components of the low-order POD modes $(k=2,3$ and 4$)$ from the velocity decomposition for the case $R e_{j}=25.8 \times 10^{3}$ and $R e_{\Omega}=2.66 \times 10^{5}$. In our POD calculation, the POD basis functions are nondimensional. The first POD mode, which reveals the structure similar to the mean flow, 

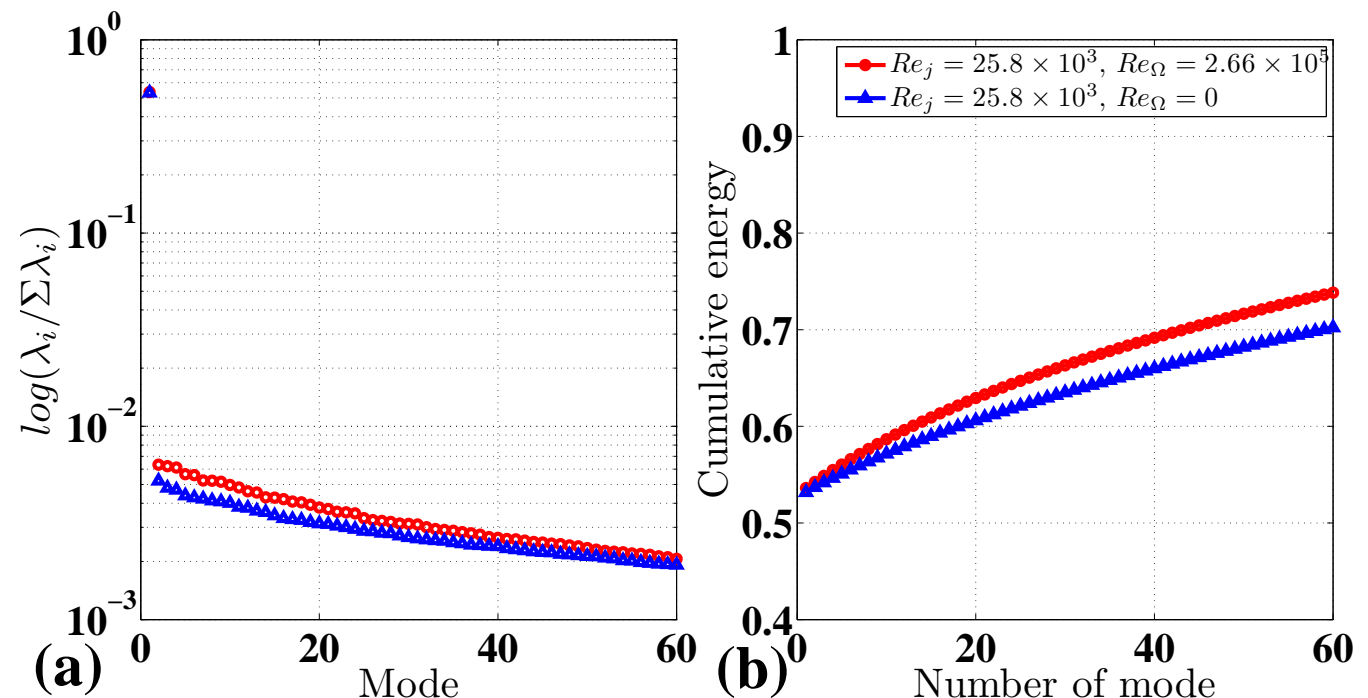

Fig. 9 POD decomposition to the PIV velocity snapshots obtained near the jet region, (a) energy spectra, (b) cumulative energy.

is not shown in figure 10, By using large-eddy simulation (LES), Hadžiabdić and Hanjalić (2008) showed that the coherent large-scale structures were created by the break down of the ring vortical structures, which are typical for a free jet. They also found that prior to the impingement, these ring vortices were deformed and tilted with respect to the impinged plane (the rotor plane in our experiment). Due to the tilting and the irregularity of the vortex breakdown, the large-scale eddies impinge on the rotor at different instants without an axial symmetry. The authors also mentioned that the asymmetric impingement of the large-scale eddy structures caused flapping around the jet axis, which enhanced the heat transfer in the jet region. In figure 10, footprints of the coherent large-scale structures discussed in Hadžiabdić and Hanjalić (2008) are displayed inside the recirculation region. Their sizes are comparable to the axial gap $e$ between the rotor and the stator. Because of the impact on the rotor, these large-scale eddies have structures that are somewhat stretched in the radial direction. The large-scale structures coexist, interact, and somewhat interconnect as they are originate from the ring vortices. However, such interactions, in combination with the shear stress caused by the rotor, increase the breaking of those structures into smaller vortical ones rolling inward and outward around the recirculation region. The eventual breakdown of the vortices and the rise in the turbulence levels near the jet region play an important role in the flow dynamics and heat transfer (Hadžiabdić and Hanialić, 2008). The formation of such roll-up vortices was also discussed in Hadžiabdić and Hanjalić (2008) as the primary and secondary vortices. Although the current PIV experiments were not able to provide the evolution of the large-scale structures, their existences can be confirmed in figure 10 .

Figure 11 shows the POD velocity decomposition for the case $R e_{j}=25.8 \times 10^{3}$ and $R e_{\Omega}=0$. Due to the absence of rotation, the flow in this case is the same as a jet impinging on a stationary surface in a very thin, confined region. Similar to figure [10, figure 11]illustrates the large-scale structures created by the breakdown of the jet ring vortices as discussed in Hadžiabdić and Hanialić (2008). These vortical structures roll outward in the wall jet; they may merge to form larger or weaker vortices, and then break into smaller-scale structures. 

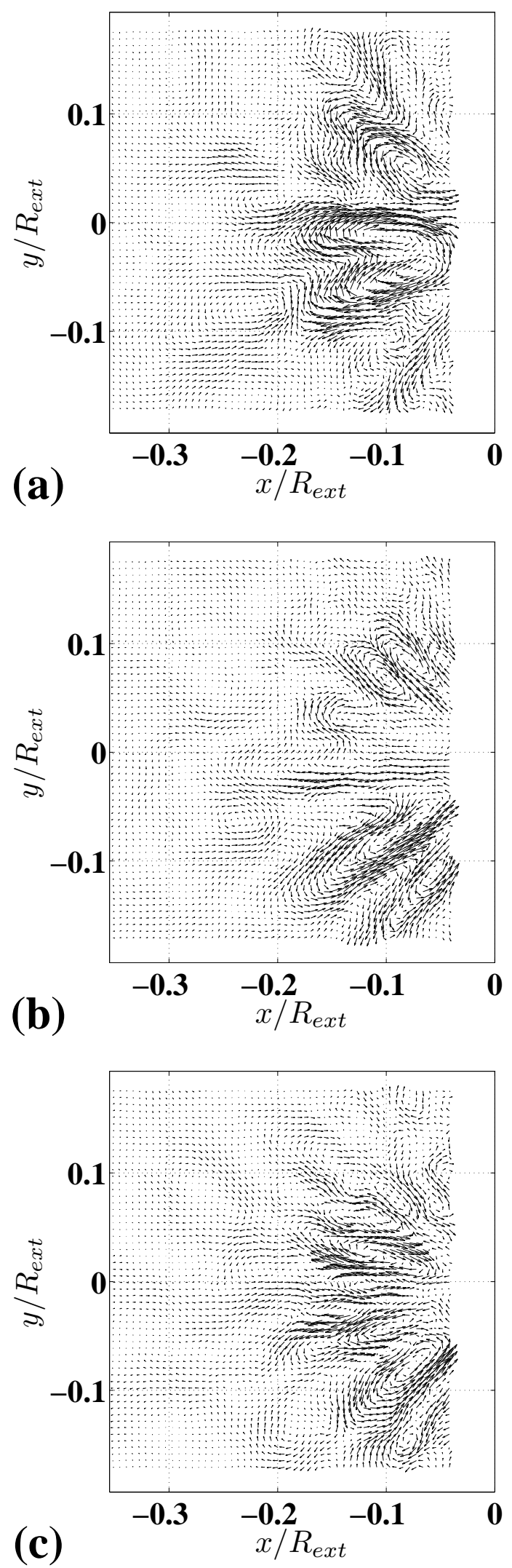

Fig. 10 Low-order POD velocity modes for the case $R e_{j}=25.8 \times 10^{3}$ and $R e_{\Omega}=2.66 \times 10^{5}$, (a) mode 2, (b) mode 3, (c) mode 4 . Vectors are de-sampled for better visibility. 

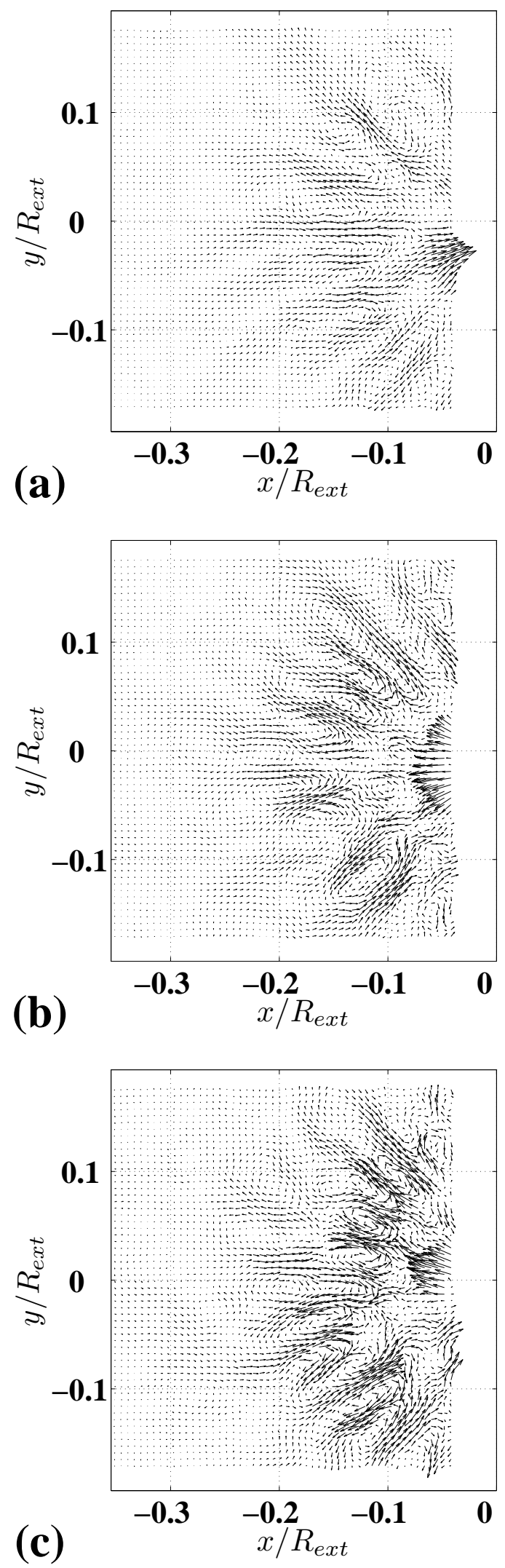

Fig. 11 Low-order POD velocity modes for the case $R e_{j}=25.8 \times 10^{3}$ and $R e_{\Omega}=0$, (a) mode 2 , (b) mode 3 , (c) mode 4 . Vectors are de-sampled for better visibility. 
The results from the POD velocity decomposition have shown that the recirculation region is observed only in the averaged flow with the presence of rotation. The kinetic energy levels contained in the low-order POD modes - the coherent large-scale structures - of the jet impinging on the rotor are higher than those of the jet impinging on the stationary surface. The large-scale structures with the same size and shape are observed in the region near the jet for the cases of the jet impinging on the surface with and without rotation.

\section{Conclusions}

In this paper, we obtained PIV measurements for an air jet impinging on an open rotor-stator system with a low non-dimensional spacing, $G=0.02$, and a very low aspect ratio, $e / D=0.25$. The velocity profiles and r.m.s fluctuating velocity distributions were obtained at three axial planes for the entire disk diameter with various rotational Reynolds numbers and jet Reynolds numbers. The PIV results were compared with those obtained with LDA measurements and an RSM simulation by Poncet et al (2005), which were carried out for a shrouded rotor-stator system that consisted of a central hub connected to the rotor and a fixed shroud. The PIV results showed that the recirculation region occurred near the jet region with the presence of the rotor. In this region, the increase of the jet velocity produced a rise in the r.m.s fluctuating velocity distributions. The background r.m.s fluctuating velocity levels nevertheless were not influenced by an increase in the rotational speed. The r.m.s fluctuating velocity reached their peaks at the radial locations $r / D$ between 1.5 and 1.6. In the open rotor-stator experiment, we also observed an increase in r.m.s fluctuating velocity near the periphery. We determined the difference in the local r.m.s fluctuating velocity peaks obtained at different axial planes. The peaks were lowest at the plane near the stator and highest at the plane near the rotor due to the transition from a laminar to a turbulent boundary layer and the growth of turbulence production caused by the near-wall shear. In addition, the radial positions of the r.m.s fluctuating velocity peaks measured at different axial planes were not identical with the peak translation of $0.25 D$, owing to the turbulence diffusion and the high shear region caused by the rotor.

Finally, we applied the POD analysis to the velocity snapshots obtained near the jet region to reveal the most dominant flow structures. The POD velocity decompositions revealed that the coherent large-scale structures possess an oval shape and their size is comparable to the axial gap spacing. For a jet impinging on a surface with and without rotation, the flow in the jet region is characterised by similar large-scale structures.

\section{Acknowledgements}

The authors are grateful for the financial support provided by the MEDEE project, the Région Nord Pasde-Calais, European project FEDER and the French National Research Agency. 


\section{References}

Baydar E, Ozmen Y (2006) An experimental investigation on flow structures of confined and unconfined impinging air jets. Heat and Mass Transfer 42(4):338-346

Berkooz G, Holmes P, Lumley J (1993) The proper orthogonal decomposition in the analysis of turbulent flows. Annual Review of Fluid Mechanics 25(1):539-575

Boutarfa R, Harmand S (2005) Local convective heat transfer for laminar and turbulent flow in a rotor-stator system. Experiments in Fluids 38(2):209-221

Brodersen S, Metzger D (1992) Experimental investigation of the flowfield resulting from the interaction between an impinging jet and a rotating disk. Experimental Thermal and Fluid Science 5(3):351-358

Coleman H, Steele W (1995) Engineering application of experimental uncertainty analysis. AIAA Journal 33(10):1888-1896

Fitzgerald J, Garimella S (1998) A study of the flow field of a confined and submerged impinging jet. International journal of heat and mass transfer 41(8-9):1025-1034

Gui L, Longo J, Stern F (2001) Towing tank PIV measurement system, data and uncertainty assessment for DTMB model 5512. Experiments in Fluids 31(3):336-346

Hadžiabdić M, Hanjalić K (2008) Vortical structures and heat transfer in a round impinging jet. Journal of Fluid Mechanics 596(1):221-260

Holmes P, Berkooz G, Lumley J (1996) Turbulence, coherent structures, dynamical systems and symmetry. Cambridge University Press, Cambridge, UK

Itoh M, Okada M (1998) An experimental study of the radial wall jet on a rotating disk. Experimental Thermal and Fluid Science 17(1-2):49-56

Lytle D, Webb B (1994) Air jet impingement heat transfer at low nozzle-plate spacings. International Journal of Heat and Mass Transfer 37(12):1687-1697

Manceau R, Perrin R, Hadziabdic M, Fourment P, Benhamadouche S (2009) Turbulent jet impinging onto a rotating disc: a collaborative evaluation of rans models. Turbulence, Heat and Mass Transfer 6

Minagawa Y, Obi S (2004) Development of turbulent impinging jet on a rotating disk. International journal of heat and fluid flow 25(5):759-766

Murray N, Ukeiley L (2007) An application of Gappy POD. Experiments in Fluids 42(1):79-91

Owen J, Rogers R (1989) Flow and heat transfer in rotating-disc systems - Vol.1: Rotor-stator systems. John Wiley and Sons Inc., New York

Pellé J, Harmand S (2009) Heat transfer study in a rotor-stator system air-gap with an axial inflow. Applied Thermal Engineering 29(8-9):1532-1543

Poncet S, Chauve M, Schiestel R (2005) Batchelor versus Stewartson flow structures in a rotor-stator cavity with throughflow. Physics of Fluids 17:075,110

Raffel M, Willert C, Wereley S, Kompenhans J (2007) Particle Image Velocimetry: a Practical Guide. Springer Verlag

Sirovich L, Kirby M (1987) Low-dimensional procedure for the characterization of human faces. Journal of the Optical Society of America A 4(3):519-524 
Westerweel J (1994) Efficient detection of spurious vectors in particle image velocimetry data. Experiments in Fluids 16(3):236-247 